\title{
Selecting the Right Plane of Dissection in Thyroidectomy: Along the Recurrent Laryngeal Nerve or the Thyroid Capsule? A Retrospective Analysis
}

\author{
Talat Waseem ${ }^{1}$ Safia Zahir Ahmed ${ }^{1} \quad$ Fatima Tuz Zahara ${ }^{1} \quad$ Muhammad Hasham Ashraf ${ }^{1}$ \\ Khwaja Muhammad Azim \\ ${ }^{1}$ Department of Surgery, Shalamar Medical and Dental College, \\ Lahore, Pakistan \\ Int Arch Otorhinolaryngol 2022;26(2):e219-e225. \\ Address for correspondence Talat Waseem, FRCSEng, FACS, DM, \\ Shalamar Medical and Dental College - Department of Surgery, \\ Lahore, Pakistan (e-mail: TWASEEM@GMAIL.COM).
}

\begin{abstract}
Keywords

- dissection

- thyroidectomy

- plane of dissection

- recurrent laryngeal nerve injury

- hypoparathyroidism

Introduction Total thyroidectomy remains highly technical, with a significant risk of recurrent laryngeal nerve (RLN) compromise and hypoparathyroidism. After identifying RLN, at the level of the ligament of Berry, local factors may compel the surgeon to either dissect along the nerve or the thyroid capsule.

Objective The objective of the present study is to compare these two approaches in terms of outcomes and complication rates.

Methods This is a retrospective analysis from September, 2013 to April 2019 of 511 consecutive patients undergoing thyroidectomy. General demographics and disease parameters were recorded. At the discretion of the surgeon and according to the demands of the local operative factors, the patients either had dissection along the RLN or along the thyroid capsule. Perioperative and postoperative parameters such as blood loss, duration of surgery, hospital stay, pain scores, analgesia requirements and complications were recorded. The groups were compared with the Pearson chi-squared test or with the Fisher exact test. A p-value $<0.05$ was considered statistically significant.

Results The incidence of transient hypocalcaemia and transient RLN compromise were higher when dissection was performed along the nerve as opposed to the plane along the thyroid capsule. Other parameters including operative time, hospital stay, pain scores, analgesia requirement, wound infection, seroma, hemorrhage, and recurrence did not differ between the groups. Subgroup analysis of the patients who presented with complications showed that local factors, malignancy, and extent of surgery correlated positively with complications when dissected along the RLN.

Conclusion Dissection along the capsule of the thyroid during thyroidectomy is a safer plane in terms of low rate of transient RLN injury and hypoparathyroidism as opposed to dissection along the nerve.
\end{abstract}

received

August 20, 2020

accepted

January 2, 2021

published online

August 23, 2021
DOI https://doi.org/ 10.1055/s-0041-1731812. ISSN 1809-9777.

\footnotetext{
(C) 2021. Fundação Otorrinolaringologia. All rights reserved.

This is an open access article published by Thieme under the terms of the Creative Commons Attribution-NonDerivative-NonCommercial-License, permitting copying and reproduction so long as the original work is given appropriate credit. Contents may not be used for commercial purposes, or adapted, remixed, transformed or built upon. (https://creativecommons.org/ licenses/by-nc-nd/4.0/)

Thieme Revinter Publicações Ltda., Rua do Matoso 170, Rio de Janeiro, RJ, CEP 20270-135, Brazil
} 


\section{Introduction}

Thyroid surgery remains a highly demanding and technical operation, despite the availability of modern technology. ${ }^{1-3}$ Postoperative hypoparathyroidism and recurrent laryngeal nerve (RLN) paralysis are still heavily feared complications, and reasons for these are multifactorial. ${ }^{4-6}$ There is a documented incidence of between 0.3 and $1.5 \%$ for permanent RLN paralysis, and of up to $10 \%$ for transient RLN compromise. ${ }^{7,8}$ Surgeon experience, quality of training, and dissection skills remain important determinants of the operative outcomes. ${ }^{9}$ Additionally, anatomical variations, functional status of the thyroid, and malignant pathology also have a certain role to play in the outcomes of this surgery. ${ }^{10-13}$

There are numerous techniques currently being practiced to dissect in this area and to avoid injury to the RLNs and to the parathyroid glands. ${ }^{11,14}$ The most common approaches are the lateral, inferior, superior, and superomedial approaches. ${ }^{15,16}$ All these techniques have their own advantages and limitations; however, there is consensus among the endocrine surgeons that dissection of the nerve around the area along the ligament of Berry can be the trickiest part of the operation. ${ }^{15}$

During the inferior and lateral approach, identification of the RLN remains a crucial step. ${ }^{16}$ At this juncture, the dissection can move either along the nerve or the capsule, but at this point, many local factors, anatomical variations, fibrosis, adhesions, and malignant invasions can all play a significant role in the choice of the plane. ${ }^{17,18}$ Remaining medially along the capsule has advantage of avoiding devascularization of the parathyroids and possibly of the nerve; however, with a higher incidence of leaving a small portion of residual thyroid tissue along the ligament of Berry. This might be acceptable for a benign pathology, but may not be for a malignant pathology in which complete resection of the thyroid tissue is the priority. On the other hand, dissection along the nerve provides dissection under vision, but makes the RLN vulnerable to traction, cautery, and devascularization injuries. ${ }^{7,8}$ This also increases the chances of devascularization of the parathyroid glands, which, in many instances, lie very close to the capsule of the gland. - Figure 1 depicts these plane choices in detail. Anatomical variations, toxicity, fibrosis or adhesions related to inflammation of a malignant pathology further add to the complexity of dissection in this area. In the present study, we evaluate the pros and cons of these two planes of dissection in clinical setting in terms of rate of complications.

\section{Patients and Methods}

Following ethical approval from the local institutional board, records of the patients undergoing thyroidectomy at a tertiary care center were reviewed. A total of 511 patients who were operated on over the course of 6 years from September, 2013 to April 2019. were included in the present study. General demographics of the patients and disease parameters were recorded. The RLN was routinely identified in all patients, and they were divided into two groups based on the dissection technique of RLN close to the ligament of Berry; in Group 1, dissection was performed along the nerve, and in Group 2, dissection was performed along the thyroid capsule. Groups 1 and 2 included 255 and 256, patients respectively. Both surgical techniques were used concurrently, and local factors guided the surgeons to opt for either technique. The present study includes the work of only two surgeons, hence minimizing variation in operational technique and performance. Neuromonitoring was not employed.

Serum calcium and parathormone (PTH) samples were drawn 48 hours after surgery. Transient postoperative hypocalcaemia was defined as single setting corrected calcium level $<8.5 \mathrm{mg} / \mathrm{dl}$ (normal: $8.5-10.5 \mathrm{mg} / \mathrm{dl}$ ). On the $1^{\text {st }}$ postoperative day, flexible fiberoptic laryngoscopy was performed
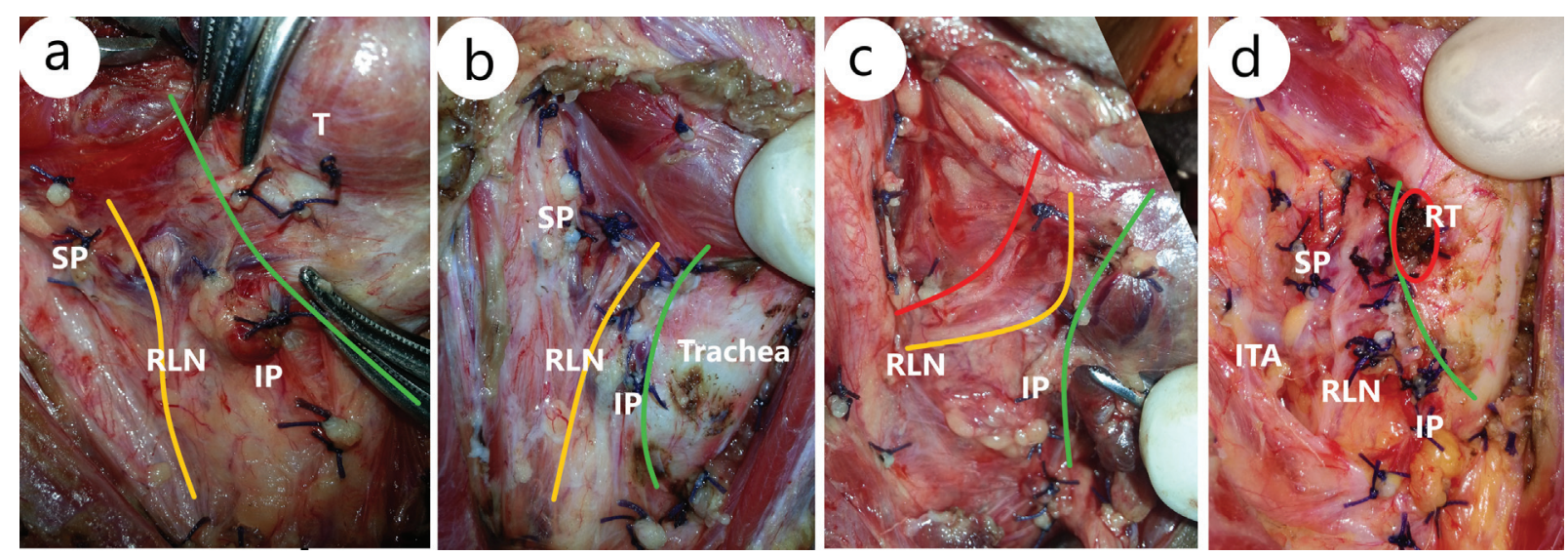

Fig. 1 The picture explains the two planes at the level of ligament of Berry, that is, a plane along the recurrent laryngeal nerve (RLN) shown by the yellow line and a tissue dissection plane which is along the thyroid capsule and medial to recurrent laryngeal nerve (shown by the green line).

-Figure 1a clearly shows that adopting a tissue dissection plane along the capsule can help in saving inferior parathyroid (IP) and superior parathyroid (SP) without devascularizing them. It also avoids traction, diathermy and manipulation injuries to the RLN. Following this technique may leave some residual thyroid tissue (RT) in few cases, as shown in - Figure 1d. Dissection along the nerve has the advantage of better exposure, which may be required in many cases to avoid injury to RLN, for example, in - Figure 1c, where there are two branches of RLN. 
Table 1 General Characteristics of the Patients participating in the study

\begin{tabular}{|c|c|c|c|}
\hline $\begin{array}{l}\text { Measurement Para- } \\
\text { meter }\end{array}$ & & $\begin{array}{l}\text { Group } 1 \\
\text { (Dissection along the nerve) } \\
(n=255)\end{array}$ & $\begin{array}{l}\text { Group } 2 \\
\text { (Dissection along the } \\
\text { thyroid capsule) } \\
(n=256)\end{array}$ \\
\hline Age (years old) & & $49.07 \pm 11.73$ & $47.96 \pm 11.13$ \\
\hline \multicolumn{4}{|l|}{ Gender } \\
\hline & Female & 207 (81.2\%) & $209(81.6 \%)$ \\
\hline & Male & $48(18.8 \%)$ & 47 (18.4\%) \\
\hline \multicolumn{4}{|l|}{ Clinical Diagnosis } \\
\hline & Follicular CA & $12(4.7 \%)$ & $20(7.6 \%)$ \\
\hline & MNG (including Toxic) & $13(5.1 \%)$ & $8(3.06 \%)$ \\
\hline & MNG involving Single Lobe & $14(5.4 \%)$ & $16(6.1 \%)$ \\
\hline & Papillary CA & $119(46.6 \%)$ & $108(41.3 \%)$ \\
\hline & Suspicious Solitary Nodule & $78(30.5 \%)$ & $83(31.8 \%)$ \\
\hline & Primary Thyrotoxicosis & $9(3 \%)$ & $12(4.5 \%)$ \\
\hline & Toxic Adenoma & $10(3.9 \%)$ & $14(5.3 \%)$ \\
\hline \multicolumn{4}{|l|}{ Histological Diagnosis } \\
\hline & Benign Follicular Lesion & $83(32 \%)$ & 87 (33\%) \\
\hline & Benign Hyperplastic Glands & $32(12 \%)$ & $34(13 \%)$ \\
\hline & Follicular Carcinoma & $16(6 \%)$ & $24(9 \%)$ \\
\hline & Papillary Carcinoma & $124(48 \%)$ & 115 (44\%) \\
\hline & Hashimoto's Thyroiditis & 0 & $1(0.3 \%)$ \\
\hline \multicolumn{4}{|l|}{ Clinical Status } \\
\hline & Euthyroid & $206(80.7 \%)$ & 201 (78.5\%) \\
\hline & Hypothyroid & $3(1 \%)$ & $2(0.7 \%)$ \\
\hline & Hyperthyroid & $46(18.03 \%)$ & $52(20.3 \%)$ \\
\hline \multicolumn{4}{|l|}{ ASA Status } \\
\hline & ASA-I & 225 (88.2\%) & $229(87.7 \%)$ \\
\hline & ASA-II & $15(5.8 \%)$ & $11(4.2 \%)$ \\
\hline & ASA-III & $12(4.7 \%)$ & $9(3.4 \%)$ \\
\hline & ASA-IV & $3(1.1 \%)$ & $7(2.6 \%)$ \\
\hline \multicolumn{4}{|l|}{$\begin{array}{l}\text { Gland Size WHO Clas- } \\
\text { sification (1974) }\end{array}$} \\
\hline & WHO Class I & $8(3.1 \%)$ & $13(4.9 \%)$ \\
\hline & WHO Class II & $7(8.75 \%)$ & $1(0.3 \%)$ \\
\hline & WHO Class III & 205 (80.4\%) & $202(77.3 \%)$ \\
\hline & WHO Class IV & $35(13.7 \%)$ & $7(2.6 \%)$ \\
\hline \multicolumn{4}{|l|}{ Type of surgery } \\
\hline & Lobectomy and Isthmectomy & $92(36.0 \%)$ & $99(37.93 \%)$ \\
\hline & Total Thyroidectomy & $136(53.3 \%)$ & $127(48.6 \%)$ \\
\hline & Completion Thyroidectomy & $8(3.1 \%)$ & $13(4.9 \%)$ \\
\hline & $\begin{array}{l}\text { Total thyroidectomy }+ \text { Neck } \\
\text { Dissection }\end{array}$ & $19(7.4 \%)$ & $17(6.5 \%)$ \\
\hline
\end{tabular}

Abbreviations: WHO, World Health Organization; CA, cancer; MNG, multinodular goiter; ASA, American Society of Anesthesiology. 
Table 2 Study endpoints summarized in both groups with and without drain

\begin{tabular}{|c|c|c|c|}
\hline Measurement parameter & $\begin{array}{l}\text { Group } 1 \\
\text { (Dissection along the nerve) } \\
(n=255)\end{array}$ & $\begin{array}{l}\text { Group } 2 \\
\text { (Dissection along the } \\
\text { thyroid capsule) } \\
(n=256)\end{array}$ & p-value \\
\hline Mean length of surgery (minutes) & $119.09 \pm 38.26$ & $117.15 \pm 41.01$ & 0.451 \\
\hline Perioperative mean blood loss & $44.72 \pm 26.03$ & $37.52 \pm 22.50$ & 0.061 \\
\hline Postoperative Drain Output & $53.22 \pm 42.98$ & $53.94 \pm 33.75$ & 0.146 \\
\hline Mean length of postoperative stay (hours) & $34.91 \pm 12.83$ & $34.69 \pm 12.75$ & 0.219 \\
\hline Mean pain score $($ maximum $=10)$ & $3.1 \pm 1.1$ & $2.3 \pm 0.4$ & 0.052 \\
\hline $\begin{array}{l}\text { Median postoperative analgesic requirements } \\
\text { according to the WHO pain ladder }\end{array}$ & Level II & Level II & 0.321 \\
\hline \multicolumn{4}{|l|}{ Complications } \\
\hline Wound infection & $8(3.1 \%)$ & $5(2 \%)$ & 0.678 \\
\hline Hematoma requiring drainage & $2(0.8 \%)$ & $1(0.4 \%)$ & 0.838 \\
\hline Seroma formation requiring drainage & $20(7.8 \%)$ & $13(5.1 \%)$ & 0.415 \\
\hline Transient hypocalcemia & $45(17.6 \%)$ & $13(5.1 \%)$ & $0.006^{*}$ \\
\hline Permanent hypocalcemia & $0 \%$ & $1(0.4 \%)$ & 0.605 \\
\hline Transient recurrent laryngeal Nerve compromise & $29(11.4 \%)$ & $3(1.2 \%)$ & $0.0001^{*}$ \\
\hline Permanent recurrent laryngeal nerve compromise & 0 & $3(1.2 \%)$ & 0.202 \\
\hline Recurrence (over a period of at least 6 months -7 years) & $2(0.8 \%)$ & 0 & 0.362 \\
\hline
\end{tabular}

Abbreviations: WHO, World Health Organization.

Means and percentages have been counted as appropriate. Mann-Whitney U t-test, Kruskal-Wallis test and Fisher exact test were applied as appropriate to find a significant difference between the groups. A $p$-value $<0.05$ was considered statistically significant.

to assess vocal cord function, if clinically indicated. Hypocalcemia and RLN compromise were considered permanent if they persisted for $>12$ months. Perioperative and postoperative surgical care parameters such as blood loss, duration of surgery, drain output, hospital stay, pain scores, analgesia requirements, and complications were reviewed and tabulated. The data were analyzed through IBM SPSS Statistics for Windows, version 21.0 (IBM Corp., Armonk, TX, USA). Means, standard deviations (SDs), and frequencies were measured as appropriate. Means were compared through the MannWhittney $\mathrm{U}$ t-test or through the Kruskal-Wallis test as statistically appropriate. The categorical data of the groups were compared with the Pearson chi-squared test ${ }^{2}$ or with the Fisher exact test as statistically appropriate. A p-value $<0.05$ was considered statistically significant.

\section{Results}

The general characteristics of the patients enrolled in the present retrospective study are summarized in -Table 1. They have been divided into two groups, and their characteristics are quite homogenous.

Surgical care parameters and complications are documented in - Table $\mathbf{2}$. The incidence of transient hypocalcemia $(p=0.006)$ and of transient RLN compromise $(p=0.0001)$ were higher when the dissection was performed along the nerve when compared with dissection performed on the plane along the thyroid capsule. A total of 58 patients developed transient hypocalcemia, 45 from Group 1 versus 13 from
Group 2. The rate of transient hypocalcemia was $17.6 \%$ in Group 1 versus 5.1\% in Group 2 ( $p<$ b0.05). Permanent hypocalcemia developed in only 1 patient of Group 2 ; however, no patient in group 1 developed permanent hypocalcemia. A total of 32 patients developed transient RLN compromise; 29 patients from Group1 and 3 patients from Group 2.

The rate of transient RLN compromise was $11.4 \%$ in Group 1 versus $1.2 \%$ in Group 2 . All three patients with permanent damage of the RLN had significant local factors; two patients had upper pole papillary tumors involving the RLN, while one suffered damage to the medial branch of the RLN during dissection.

Other complications, including operative time, hospital stay, pain scores, analgesia requirement, wound infection, seroma, hemorrhage and recurrence did not differ between the groups.

The subgroup analysis of the patients who presented with complications showed that malignancy and extent of surgery correlated positively with complications when the dissection was performed along the RLN, as summarized in - Table 3. Papillary carcinoma and higher extent of operative dissection in form of total thyroidectomy positively correlated with higher chances of postoperative hypoparathyroidism and RLN compromise; however, considering the sample size of the subgroups, this data should only form a basis for further enquiry on these parameters.

\section{Discussion}

Preventing RLN injuries and devascularization of parathyroid glands is a technically daunting surgical skill that depends on 


\begin{tabular}{|c|c|c|c|c|c|c|c|c|c|c|c|c|c|}
\hline & 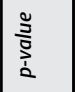 & 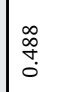 & I & 1 & $\mid \begin{array}{l}0 \\
\text {. } \\
0\end{array}$ & । & $\begin{array}{l}\text { 酋 } \\
\text { d. }\end{array}$ & 1 & 1 & 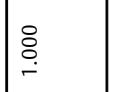 & $\left|\begin{array}{c}\bar{b} \\
0 \\
0\end{array}\right|$ & & । \\
\hline & 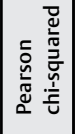 & 苔 & I & I & $\begin{array}{l}\mathscr{O} \\
\stackrel{\circ}{\circ} \\
0\end{array}$ & I & $\begin{array}{l}\stackrel{\sim}{\infty} \\
\stackrel{\leftrightarrow}{-}\end{array}$ & 1 & 1 & 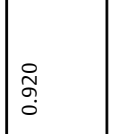 & $\left|\begin{array}{r}\hat{\tilde{p}} \\
\stackrel{i}{i}\end{array}\right|$ & 1 & 1 \\
\hline & 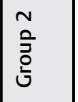 & $\mid \begin{array}{c}\widehat{\widehat{\alpha}} \\
0 \\
0\end{array}$ & 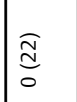 & 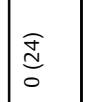 & $\begin{array}{l}\widehat{\sigma} \\
\stackrel{\Xi}{\Xi} \\
0\end{array}$ & $\begin{array}{l}\widehat{\widehat{N}} \\
0 \\
0\end{array}$ & 恿 & $\begin{array}{l}\widehat{\tilde{m}} \\
0\end{array}$ & 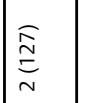 & $\mid \begin{array}{l}E \\
\stackrel{E}{0}\end{array}$ & $\mid \begin{array}{c}\widehat{a} \\
\grave{d} \\
0\end{array}$ & $\mid \begin{array}{l}\widehat{a} \\
0\end{array}$ & 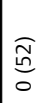 \\
\hline 离 & \begin{tabular}{|l}
$\overline{\frac{2}{3}}$ \\
产
\end{tabular} & $\frac{\widehat{\infty}}{\stackrel{\infty}{\infty}}$ & $\begin{array}{l}\widehat{\widehat{d}} \\
0\end{array}$ & $\begin{array}{l}0 \\
\stackrel{0}{0} \\
0\end{array}$ & 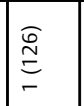 & बa & $\mid \begin{array}{l}\widehat{\widehat{\sigma}} \\
0 \\
0\end{array}$ & $\frac{\infty}{0}$ & \begin{tabular}{|l}
$\widehat{O}$ \\
$\stackrel{0}{0}$ \\
0
\end{tabular} & $\mid \begin{array}{l}\widehat{a} \\
\stackrel{0}{0}\end{array}$ & 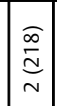 & 商 & 夏 \\
\hline & 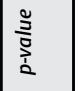 & 1 & 1 & 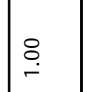 & $\begin{array}{c}\stackrel{y}{m} \\
\stackrel{m}{0}\end{array}$ & 1 & 1 & 1 & $\begin{array}{l}\stackrel{m}{0} \\
\stackrel{0}{0}\end{array}$ & $\mid \begin{array}{l}\tilde{N} \\
\tilde{0} \\
0\end{array}$ & $\left|\begin{array}{l}0 \\
\grave{2} \\
0\end{array}\right|$ & & , \\
\hline | & 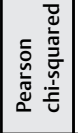 & 1 & 1 & $\begin{array}{l}\mathbf{a} \\
0 \\
0 \\
0\end{array}$ & $\frac{\stackrel{g}{i}}{i}$ & 1 & 1 & 1 & $\frac{\mathscr{Q}}{\stackrel{i}{i}}$ & $\stackrel{\stackrel{\rho}{\mathscr{n}}}{=}$ & $\mid \begin{array}{l}n \\
\tilde{m} \\
\tilde{m}\end{array}$ & & । \\
\hline 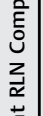 & $\begin{array}{l}\text { N } \\
\text { 言 } \\
\text { ปे }\end{array}$ & $\underset{\substack{\widehat{\infty} \\
0}}{\widehat{c}}$ & 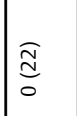 & $\underset{\sim}{\stackrel{\mathcal{I}}{\mathcal{I}}}$ & \begin{tabular}{|l}
$\underline{\varrho}$ \\
$\stackrel{5}{0}$ \\
0
\end{tabular} & $\begin{array}{l}\widehat{I} \\
\stackrel{\Xi}{0} \\
0\end{array}$ & $\mid \begin{array}{l}\widehat{\widehat{g}} \\
0\end{array}$ & $\frac{\widehat{m}}{\tilde{y}_{0}}$ & 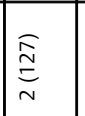 & $\underset{E}{\stackrel{E}{E}}$ & $\mid \begin{array}{l}\mathbb{\Omega} \\
\stackrel{d}{d} \\
0\end{array}$ & à & 可 \\
\hline 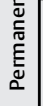 & 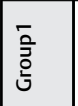 & $\stackrel{\substack{\infty \\
0}}{0}$ & $\begin{array}{l}\widehat{\widetilde{\vartheta}} \\
0\end{array}$ & $\begin{array}{l}0 \\
\stackrel{0}{0} \\
0\end{array}$ & 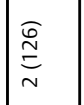 & ब⿳亠口冋口 & $\begin{array}{l}\widehat{\widetilde{g}} \\
0\end{array}$ & $\frac{\widehat{\infty}}{0}$ & $\mid \begin{array}{l}\widehat{\infty} \\
\stackrel{\infty}{n} \\
0\end{array}$ & $\frac{\widehat{\Omega}}{\mathfrak{O}}$ & \begin{tabular}{|l|}
$\widehat{\infty}$ \\
$\stackrel{\infty}{d}$ \\
$m$
\end{tabular} & 商 & 高 \\
\hline & 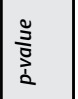 & 立 & $\frac{9}{0}$ & $\mid \begin{array}{l}0 \\
⿱ 亠 ⿱ 十 冖 \\
0\end{array}$ & $\begin{array}{l}\hat{f} \\
0 \\
0\end{array}$ & $\stackrel{\leftrightarrow}{\circ}$ & 蒿 & 1 & \begin{tabular}{|l}
$\stackrel{*}{*}$ \\
$\stackrel{0}{0}$ \\
$\dot{0}$
\end{tabular} & 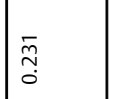 & $\mid \begin{array}{l}0 \\
\vdots \\
0 \\
0 \\
0\end{array}$ & $\underset{-}{\stackrel{\circ}{-}}$ & $\mid \begin{array}{l}\infty \\
0 \\
0 \\
0 \\
0\end{array}$ \\
\hline 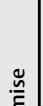 & 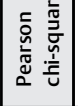 & $\begin{array}{l}n \\
\hat{0} \\
0 \\
0 \\
-\end{array}$ & $\mid \frac{g}{\dot{\sigma}}$ & 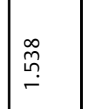 & $\frac{\hat{m}}{\omega}$ & $\mid \begin{array}{c}0 \\
\substack{\infty \\
0 \\
0}\end{array}$ & \begin{tabular}{|l}
0 \\
$\infty$ \\
$\infty$ \\
0 \\
\hdashline
\end{tabular} & 1 & \begin{tabular}{|c}
0 \\
$\mathscr{m}$ \\
$\infty$ \\
$\infty$
\end{tabular} & $\begin{array}{l}\stackrel{\infty}{\sim} \\
\stackrel{\sim}{\sim}\end{array}$ & 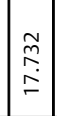 & \begin{tabular}{|c|c|}
$\tilde{c}$ \\
$\infty$ \\
0 \\
0 \\
\end{tabular} & 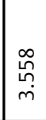 \\
\hline 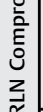 & 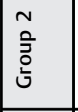 & $\stackrel{\sqrt{\infty}}{\stackrel{\widehat{\infty}}{-}}$ & $\underset{\substack{\mathbb{N} \\
0}}{\mathbb{D}}$ & 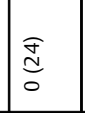 & $\underset{\sim}{\stackrel{O}{\Xi}}$ & 仝 & $\stackrel{\text { g }}{\sigma}$ & $\underset{0}{\widehat{m}}$ & $\underset{\substack{\widehat{N} \\
\sim}}{\stackrel{N}{N}}$ & $\underset{O}{E}$ & $\begin{array}{l}\widehat{E} \\
\stackrel{a}{d} \\
m\end{array}$ & $\widehat{\widehat{a}}$ & 芯 \\
\hline 竧 & 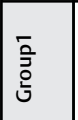 & $\stackrel{\widehat{\widetilde{x}}}{\stackrel{\widehat{m}}{\simeq}}$ & $\underset{\widetilde{\widetilde{d}}}{\widehat{\widetilde{\jmath}}}$ & 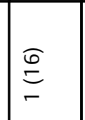 & $\begin{array}{l}\stackrel{\widehat{O}}{D} \\
\stackrel{D}{\Xi} \\
=\end{array}$ & $\frac{\widehat{O}}{-}$ & $\begin{array}{l}\widehat{\widetilde{\sigma}} \\
\stackrel{N}{\simeq}\end{array}$ & $\frac{\infty}{0}$ & 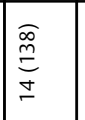 & $\stackrel{\widehat{O}}{\stackrel{g}{m}}$ & 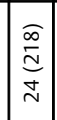 & $\widehat{\underline{m}}$ & $\frac{\sigma}{\sigma}$ \\
\hline & 愛 & , & $\mid \begin{array}{l}\infty \\
\frac{\infty}{0}\end{array}$ & $\underset{-}{\stackrel{\leftrightarrow}{\circ}}$ & | & $\begin{array}{l}\stackrel{n}{f} \\
f \\
0\end{array}$ & 1 & $\frac{8}{0}$ & 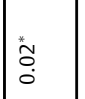 & 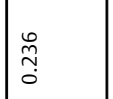 & \begin{tabular}{|l}
0 \\
$\vdots$ \\
0 \\
0 \\
0
\end{tabular} & & $\begin{array}{l}\infty \\
0 \\
0 \\
0\end{array}$ \\
\hline & 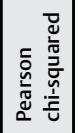 & 1 & $\mid \begin{array}{l}\hat{N} \\
\infty \\
i\end{array}$ & 命 & $\begin{array}{l}\stackrel{g}{\circ} \\
\stackrel{\alpha}{\circ}\end{array}$ & $\stackrel{\infty}{\stackrel{\infty}{\stackrel{2}{\sim}}}$ & , & 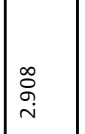 & 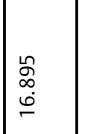 & $\begin{array}{l}\infty \\
\stackrel{\infty}{\sim} \\
\stackrel{\sim}{i}\end{array}$ & 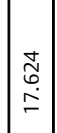 & & \begin{tabular}{|l}
$\stackrel{\leftrightarrow}{\tilde{\omega}}$ \\
$\stackrel{\rho}{n}$
\end{tabular} \\
\hline 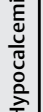 & $\begin{array}{l}N \\
\vdots \\
\vdots \\
\vdots \\
\vdots\end{array}$ & $\underset{\substack{\infty \\
0}}{\widehat{\infty}}$ & $\underset{\sim}{\stackrel{\mathbb{N}}{\sim}}$ & $\underset{\sigma}{\stackrel{\mathcal{V}}{d}}$ & $\mid \begin{array}{c}\sigma \\
ٍ \infty \\
\infty\end{array}$ & 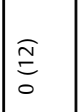 & 骨 & $\underset{\sim}{\stackrel{N}{N}}$ & 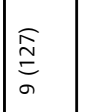 & $\underset{\sim}{\stackrel{E}{E}}$ & 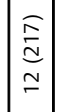 & $\widehat{\widehat{a}}$ & 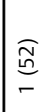 \\
\hline 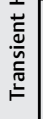 & - & $\stackrel{\widehat{\infty}}{\stackrel{\infty}{0}}$ & 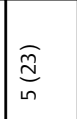 & $\underset{m}{\stackrel{\sigma}{m}}$ & 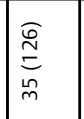 & $\underset{\sim}{\stackrel{\widehat{O}}{\sim}}$ & 胥 & $\frac{\widehat{\infty}}{\sigma}$ & 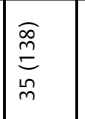 & $\underset{0}{\stackrel{9}{O}}$ & \begin{tabular}{|c|}
$\widehat{\infty}$ \\
$\frac{\infty}{d}$ \\
$\dot{q}$
\end{tabular} & $\widehat{\widehat{m}}$ & 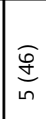 \\
\hline & & 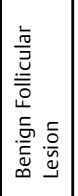 & 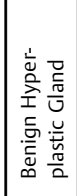 & 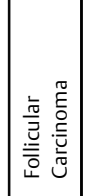 & 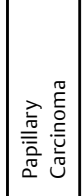 & 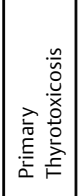 & 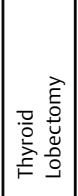 & 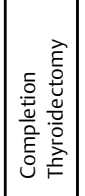 & 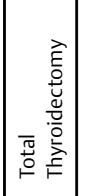 & 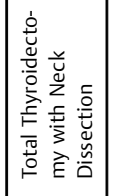 & 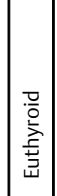 & 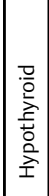 & 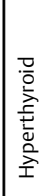 \\
\hline 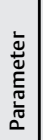 & & 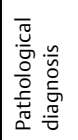 & & & & & 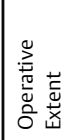 & & & & 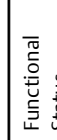 & & \\
\hline
\end{tabular}


many factors, including surgeon experience, volume of surgery, and quality of the training of the endocrine surgeon. $8,10,19$ Anatomical variations make process of dissection even more challenging. ${ }^{15,20}$ The ability of the surgeon to recognize and adapt to anatomical variations remains the most important determinant of overall outcome of the operation. Moreover, fibrosis, adhesions and malignant invasion have their own bequest to add up to this complexity. $^{21,22}$

As shown in the present study, the plane of dissection can enormously influence the outcome of the procedure. Many parathyroid glands lie in close association with the thyroid capsule, especially the inferior ones, which may be saved more reliably if dissected along the capsule. ${ }^{23}$ Similarly, the incidence of injuries may be intuitively lessened if the dissection is performed along the capsule. The data of the present study corroborates this intuitive notion. In certain circumstances, it may become quite difficult to dissect along the capsule, or the local pathology may dictate the surgeon to dissect along the nerve. Here, the value of intraoperative nerve monitoring comes to focus. ${ }^{20,24,25}$

Transient RLN compromise has been traditionally underreported. Despite a very careful dissection, the incidence of neuropraxia leading to transient RLN compromise remains quite high in our study, of up to $11 \%$ if dissected along the nerve, and of $\sim 5 \%$ if dissected along the capsule. ${ }^{26}$ The nerve injuries are related to traction, cautery, and dissectionrelated devascularization. No patient in our study exhibited bilateral paralysis. The decision in favor of dissection along the thyroid capsule is straightforward in case of a benign pathology; however, in many instances, the dissection becomes difficult along the capsule, and it may have to be opted to dissect along the nerve.

Inherently, the dissection along the thyroid capsule has many disadvantages. The surgeon may have to rely on the projected course of RLN and would naturally be inclined to leave a small sleeve of thyroid tissue. This loss of clarity about the nerve along the ligament of Berry can put the nerve at higher risk. Moreover, if the surgeon leaves a sleeve of thyroid tissue while saving the nerve, this may lead to inadequate resection, especially when dealing with a malignant gland. Similarly, the tumor involving the upper pole and the ligament of Berry area can be very tricky regardless of the chosen plane of dissection, since, at times, there is complete loss of the planes.

It remains imperative to understand why dissection along the nerve is potentially more harmful for the patient. There can be many plausible explanations, of which one is the devascularization and manipulation of the nerves and of the parathyroid glands during dissection. ${ }^{26}$ Other reasons could be the adhesions related to redo surgery, radiation, of fibrosis due to some inflammatory condition like thyroiditis or malignant invasion itself. ${ }^{17}$ The subgroup analysis of the present study clearly points towards these plausible reasons.

The present study has many limitations. It is a retrospective analysis, and many factors play a role as the patient develops these complications. The subgroup analysis needs to be interpreted very carefully and would require further confirmation through clinical trials.

\section{Conclusion}

In conclusion, dissection along the capsule of the thyroid during thyroidectomy appears to be a safer plane in terms of low complication rate as opposed to dissection along the nerve. It may be employed increasingly, especially for benign pathologies of thyroid gland.

\section{Conflict of Interests}

The authors have no conflict of interest to declare.

\section{Acknowledgement and Disclaimers}

The authors acknowledge the support of the Department of Surgery and its staff in conducting and managing the present scientific work. The authors disclose no financial and direct or indirect personal relationships with other people or organizations that could potentially and inappropriately influence (bias) the present work and conclusions. No additional funding was required for this particular work.

Total thyroidectomy still remains highly technical with significant risk of RLN compromise and hypoparathyroidism.

While identifying RLN, the surgeon can either dissect along the nerve or the thyroid capsule.

In the present study, we show that dissection along the capsule of the thyroid during thyroidectomy is safer in terms of low rate of transient RLN injury and hypoparathyroidism.

\section{References}

1 Hannan SA. The magnificent seven: a history of modern thyroid surgery. Int J Surg 2006;4(03):187-191

2 Seybt MW, Terris DJ. Outpatient thyroidectomy: experience in over 200 patients. Laryngoscope 2010;120(05):959-963http:// www.ncbi.nlm.nih.gov/pubmed/20422690. Cited 2020 Jun 17

3 Conzo G, Avenia N, Bellastella G, et al. The role of surgery in the current management of differentiated thyroid cancer. Vol. 47. Endocrine. Humana Press Inc.; 2014:380-8

4 Duclos A, Peix JL, Colin C, et al; CATHY Study Group. Influence of experience on performance of individual surgeons in thyroid surgery: prospective cross sectional multicentre study. BMJ 2012;344(7843):1-11

5 Sarı S, Erbil Y, Sümer A, et al. Evaluation of recurrent laryngeal nerve monitoring in thyroid surgery. Int J Surg 2010;8(06): 474-478

6 Lee MS, Lee UY, Lee JH, Han SH. Relative direction and position of recurrent laryngeal nerve for anatomical configuration. Surg Radiol Anat 2009;31(09):649-655

7 Hayward NJ, Grodski S, Yeung M, Johnson WR, Serpell J. Recurrent laryngeal nerve injury in thyroid surgery: a review. ANZ J Surg 2013;83(1-2):15-21

8 Serpell JW, Lee JC, Yeung MJ, Grodski S, Johnson W, Bailey M. Differential recurrent laryngeal nerve palsy rates after thyroidectomy. Surgery 2014;156(05):1157-1166

9 Tsai S-H, Chien S-C, Nguyen P-A, et al. Incidences of Hypothyroidism Associated With Surgical Procedures for Thyroid Disorders: A 
Nationwide Population-Based Study. Front Pharmacol 2019; 10:1-8

10 Kaisha W, Wobenjo A, Saidi H. Topography of the recurrent laryngeal nerve in relation to the thyroid artery, Zuckerkandl tubercle, and Berry ligament in Kenyans. Clin Anat 2011;24(07): 853-857

11 Antakia R, Edafe O, Uttley L, Balasubramanian SP. Effectiveness of preventative and other surgical measures on hypocalcemia following bilateral thyroid surgery: a systematic review and metaanalysis. Thyroid 2015;25(01):95-106

12 Gray SW, Skandalakis JE, Akin JT Jr. Embryological considerations of thyroid surgery: developmental anatomy of the thyroid, parathyroids and the recurrent laryngeal nerve. Am Surg 1976;42(09): 621-628

13 Rulli F, Ambrogi V, Dionigi G, et al. Meta-analysis of recurrent laryngeal nerve injury in thyroid surgery with or without intraoperative nerve monitoring. Acta Otorhinolaryngol Ital 2014;34 (04):223-229

14 Aytac B, Karamercan A. Recurrent laryngeal nerve injury and preservation in thyroidectomy. Saudi Med J 2005;26(11): 1746-1749

15 Page C, Peltier J, Charlet L, Laude M, Strunski V. Superior approach to the inferior laryngeal nerve in thyroid surgery: anatomy, surgical technique and indications. Surg Radiol Anat 2006;28 (06):631-636

16 Sreejayan MP, Arun S, Jose J. Inferior Approach: a Safe Method for Identification of Recurrent Laryngeal Nerve During Thyroidectomy. Indian J Surg 2019;81(05):474-478

17 Patoir A, Payet C, Peix J-L, et al; CATHY Study Group. Determinants of operative time in thyroid surgery: A prospective multicenter study of 3454 thyroidectomies. PLoS One 2017;12(07):1-11. Available from: https://dx.plos.org/10.1371/journal.pone.0181424
18 Hemmaoui B, Bouaiti EA, Sahli M, et al. [The inferior laryngeal nerve: anatomical and surgical considerations about 60 thyroidectomy]. Pan Afr Med J 2019;33:1-5

19 D'Orazi V, Sacconi A, Trombetta S, et al. May predictors of difficulty in thyroid surgery increase the incidence of complications? Prospective study with the proposal of a preoperative score. BMC Surg 2019;18(Suppl 1):1-8

20 Wong KP, Mak KL, Wong CKH, Lang BHH. Systematic review and meta-analysis on intra-operative neuro-monitoring in high-risk thyroidectomy. Int J Surg 2017;38:21-30

21 Vieni S, Graceffa G, Rizzo GEM, Latteri F, Latteri MA, Cipolla C. An evaluation score of the difficulty of thyroidectomy considering operating time and preservation of recurrent laryngeal nerve. Updates Surg 2019;71(03):569-577

22 Chiang FY, Wang LF, Huang YF, Lee KW, Kuo WR. Recurrent laryngeal nerve palsy after thyroidectomy with routine identification of the recurrent laryngeal nerve. Surgery 2005;137(03): 342-347

23 Thomusch O, Machens A, Sekulla C, Ukkat J, Brauckhoff M, Dralle $\mathrm{H}$. The impact of surgical technique on postoperative hypoparathyroidism in bilateral thyroid surgery: a multivariate analysis of 5846 consecutive patients. Surgery 2003;133(02):180-185

24 Dralle H, Sekulla C, Lorenz K, Brauckhoff M, Machens AGerman IONM Study Group. Intraoperative monitoring of the recurrent laryngeal nerve in thyroid surgery. World J Surg 2008;32(07): 1358-1366

25 Liu JB, Sosa JA, Grogan RH, et al. Variation of thyroidectomy-specific outcomes among hospitals and their association with risk adjustment and hospital performance. JAMA Surg 2018;153(01):1-10

26 Serpell JW, Lee JC, Chiu WK, Edwards G. Stressing the recurrent laryngeal nerve during thyroidectomy. ANZ J Surg 2015;85(12): 962-965 